\title{
Arithmetization and rigor as beliefs in the development of mathematics
}

\author{
Lorena Segura and Juan Matías Sepulcre \\ Department of Mathematical Analysis, University of Alicante, 03080-Alicante, \\ Spain
}

\begin{abstract}
With the arrival of the 19th century, a process of change guided the treatment of three basic elements in the development of mathematics: rigour, the arithmetization and the clarification of the concept of function, categorised as the most important tool in the development of the mathematical analysis. In this paper we will show how several prominent mathematicians contributed greatly to the development of these basic elements that allowed the solid underpinning of mathematics and the consideration of mathematics as an axiomatic way of thinking in which anyone can deduce valid conclusions from certain types of premises. This $19^{\text {th }}$ century stage shares, possibly with the Heroic Age of Ancient Greece, the most revolutionary period in all history of mathematics.
\end{abstract}

Keywords Mathematics · Rigour · Arithmetization · Analysis · Function

\section{Introduction}

The Pythagorean school, founded by Pythagoras in the $5^{\text {th }}$ Century B.C, focussed on studying natural and their relationships, as the existence of mathematical connections in many phenomena of Nature (such as Astronomy or Music) was a confirmable fact. This study led to the development of an important part of arithmetic.

Much later, starting in the 13th Century, an intellectual renaissance took place in the West as a result of the Catholic Church's acceptance of Greek culture and Aristotelian thought, whilst from the 14th Century on, there was a real demand for more accurate methods for: the search for new materials, the development of technology, the accurate calculation of positions at sea (following geographical exploration that covered great distances in open sea), the improvement of mechanisms of calculus (stimulated by the development of trade) or the solving of problems of a military nature brought about by the introduction of gunpowder (such as the movement and trajectory of shells).

At the same time, the discovery of other civilisations had liberalising consequences for European culture and the printing press encouraged the wide dissemination of knowledge. All these reasons led to the fact that, in modern European civilisation, priority was given to the search for knowledge and scientific development, for the purpose of explaining the phenomena of Nature. This was the spirit that was made evident in Leopoldo Varela's quote "The absence of doubt leads Man to an absence of curiosity and then there would be no inquisitiveness. Therefore no mathematics" (Ortega 2002). 
During the Renaissance, mathematicians expressed more interest in producing new methods and results than in the rigour of the demonstrations. This, together with the emergence of a new adapted symbolic script, made it much easier to develop new formal calculus techniques and infinitesimal methods. This course of action was reflected years later in the surprising quote from Bonaventura Cavalieri: "Rigour is the concern of philosophers, rather than of mathematicians", included in the work of Fernando Bombal (Bombal 2010).

So, generally, in the ordinary mathematical works prior to the 18th Century, the only steps that appeared were those that entailed the introduction of some new aspect or original idea. Nevertheless, the time came when the more clearsighted spirits began to question the consistency of their constructions. In fact, the necessity arose of formally demonstrating all the results that were incorporated, even though it is true that the idea of formal and rigorous demonstration would change depending on the context and the cultural environment. During the 18th Century mathematical interests centred on putting forward predictions with high doses of certainty, backed up by verifying the result in a series of specific cases and supported by numeric examples that provided reliability for what had been stated. This gave rise to different controversies between the mathematicians of the period, who questioned the acceptance of the new results. George Berkeley's publication in 1734 of The Analyst, should be mentioned as an example that constitutes a criticism of the fundamentals of science, a very influential work in the development of mathematics (Berkeley 1734).

All of this, at the beginning of the 19th Century, resulted in a profound review of Calculus. Also, during the rigorization process that continued during the whole of the 19th Century, the very notion of rigour was changing, as the consensus on what is a trivial step within a test depends as well on the historical and cultural context (Struik 1986).

Carl Friedrich Gauss's work Disquisitiones Arithmeticae (Gauss 1863) is considered as one of the first works to represent mathematic rigour. It is written completely in the style of theorem-demonstration-corollary and at no point does it reveal the causes that motivated him to follow specific lines of development of the demonstrations.

In this work we will set out some of the steps taken along this path towards the rigorization of mathematics. Initially we will put special emphasis on the triggering of this process and on the representative concept of function, which was one of the issues that had to be reviewed due to the emergence of a large number and variety of functions in the activity of the mathematicians of the period. We will conclude with the arithmetization of analysis and we will express some reflections on the problem unleashed by the great number of mathematical results produced, which makes it impossible for the specialists to have knowledge and command over all these advances. 


\section{Beginning of the process}

The search for consistency in the mathematical constructs became a necessity for admitting the new results that were coming to light. This sensation of a lack of consistency and rigour led to a constant source of paradoxes and contradictions, giving rise to many controversies and a great sensation of uncertainty. With this the process of rigorization began, which contributed enormously to the fact that, in Europe, the 18th and 19th Centuries were considered as the Golden Age of Analysis and, more specifically, the 19th Century was considered by many mathematics historians, such as Carl Boyer (Boyer 1986), as the Golden Age of Mathematics.

In particular, in the early 19th Century, the figure of Augustin Louis Cauchy stands out, who during the first twenty years of that century took the concepts of limit and continuity as the organising elements of his programme (Montesinos y Valdivia 2000). In his Cours d'Analyse (Cauchy 1821), Cauchy states and proves that the sum of a convergent sequence of continuous functions defines a continuous function. But in 1826 Niels Henrik Abel (Abel 1826, pp. 224-225) proposed a Fourier series as a counter-example to this assertion, which converges to a discontinuous function, furthermore indicating that "Cauchy's theorem appears to admit some exceptions.." even though he does not show the error committed by the author in his demonstration. This type of situation was regularly repeated, provoking throughout the 19th Century a process for the search for a rigorous, formal substantiation of mathematics and, more specifically, of mathematical analysis.

Bernhard Bolzano had already shown himself in his work of 1817 (Bolzano 1817) to be clearly aware of the need for rigour in analysis, which led Felix Klein to consider him as the father of arithmetization. Nevertheless, at that time, Bolzano had little influence with the mathematics community as Cauchy's analysis, strongly conditioned by geometric intuition, still persisted. In addition, around 1830, Bolzano had made an attempt to develop a theory of real numbers as limits of sequences of rational numbers, but this work went unnoticed and was not published until 1962.

The sensation of uncertainty in the results, together with the appearance of new theories such as non-Euclidean Geometry, generated the need to clearly and accurately establish those properties of the objects under study. They chose to return to the axiomatic method used by the Greeks in the development of Euclidean geometry. In fact, there was a substantial change from a distinctly geometric conception to a more algebraic-analytic treatment. Other mathematicians who stand out by forming part of this change were Leonhard Euler and Joseph-Louis Lagrange.

The impact of this new precision in the definition of concepts was huge. Only that which could be defined in terms of well-established earlier structures was acceptable. In reality, this growing enthusiasm for precision in the outcome in itself bore the seed of an increasingly retroactive nature.

A process began of questioning all structures which were traditionally considered as valid and well established. Nothing escaped rigorous analysis, 
precise formulation, and stringent justification. Mathematics became synonymous with proof; without proof there was nothing. They even looked in depth into the need to prove the existence of the mathematical entities used, which then ceased to be seen as unquestionable truths due to their heuristic nature and their original geometry basis. In this way, taking into account the invalidation of many results that were incorrectly incorporated, all trace of subjectivity still existing in the demonstrations was removed.

\section{Concept of function}

In the 18th and 19th Centuries, when mathematical analysis definitively became a part of the scientific panorama, functions were considered as the objects par excellence of mathematics (Rey Pastor \& Babini 1997).

Function is undoubtedly the key word in analysis, and it was precisely in the clarification of this term that the tendency arose for the arithmetization of analysis, a term coined by Felix Klein in 1895 (Boyer 1986, p. 685).

The Babylonian mathematicians already intuitively handled the concept of function, an aspect that was observed in the specific studies of Astronomy, when they turned to tables of data, interpolation and extrapolation in the search for patterns and proportions. The Greek mathematicians also made use of this concept through the proportions that enabled the comparison of variables in Nature itself. In the $17^{\text {th }}$ Century, René Descartes and Pierre de Fermat established connections between Geometry and Algebra, taking the first steps towards Analytic Geometry, in which the relationship of dependence between two variable quantities is explicitly stated and the concept of function is associated to a curve representing the path of moving points.

The curious behaviour of a vibrating string generated great interest amongst mathematicians, leading to one of the most heated and productive disputes in the history of mathematics, which is discussed in detail in (Ferreirós 2003). Until the 18th Century, mathematics had not developed sufficiently to be able to tackle this problem such as it is discussed in (Boyer 1986). It was in 1715 when Brook Taylor affirmed that the movement of an arbitrary point on the string is the same as a simple pendulum and, therefore, the form of the curve adopted by the piece of string at any given moment should be sinusoidal. The fundamental sound corresponding to that pendulum vibration is not the only sound emitted by the vibrating string, as other, less intense sounds appear simultaneously. What is really curious is the fact that the string does not change alternately from one overtone to another, but it emits all the harmonic sounds concurrently. This fact caused great intrigue and discussion amongst the mathematicians of the period. Several great mathematicians like Jean le Rond d'Alembert, Leonhard Euler, Daniel Bernoulli or Joseph Fourier tried to explain this question.

The attempts to provide a solution to this problem gave rise to differences of opinion on how to represent functions. The problem implied the generalisation of the concept of function in order to establish a one-to-one correspondence between functions and curves. D'Alembert and Euler used a pair of arbitrary 
functions, not necessarily periodic, whilst Bernoulli gave the solution in terms of a finite series of trigonometric functions. The mathematics community seems to have considered Bernoulli's answer as being less general than that put forward by d'Alembert and Euler, until Fourier entered the scene in 1824 with a double contribution. On the one hand by demonstrating that Bernoulli was right, and on the other, by making one of the most important contributions to mathematics. To be specific, Fourier demonstrated that any $y=f(x)$ function can be represented by a series, which in his honour was known as a Fourier series, of the form

$$
f(x)=\frac{a_{0}}{2}+\sum_{n=1}^{\infty}\left(a_{n} \cos (n x)+b_{n} \sin (n x)\right) .
$$

In comparison with the Taylor series, functions represented using the Fourier series allow for a much higher degree of generalisation. Even in the event that the function has many points where there is no derivative or in which the function is not continuous, it can also be developed under the Fourier series. Therefore, functions no longer needed to be as well behaved in their form as those that mathematicians had been working with up until then. For example, for Euler, discontinuous functions in general could not be expressed analytically and consequently the definition that had initially been used was too restrictive to provide a solution to this problem of the vibrating string.

So, the concept of function was not clarified up until that moment. For Carl Friedrich Gauss a function was a closed finite analytical expression, even though he referred to hypergeometric series as functions, but without the total conviction that they could be regarded as functions. Similarly, Sylvestre François Lacroix stated that "Every quantity whose value depends on one or several others is called a function of the latter, whether one knows or does not know by what operations it is necessary to go from the latter to the first quantity" (Lacroix 1797, p. 1).

For his part, Lejeune Dirichlet put forward in 1837, in his work Über die Darstellung ganz willkürlicher Functionen durch Sinus und Cosinus-reihen (Mannheim 1964, p. 52), a definition of function that was fairly broad and general: «If a variable $y$ is associated with another variable $x$ in such a way that $x$ is always attributed a numerical value, there is a rule by which a unique value of $y$ is determined, and then it can be said that $y$ is a function of the independent variable $x$ ». As we can see, this definition comes much closer to the modern idea of a general correspondence between two sets of real numbers.

In this respect, we should also highlight the emphasis given by Euler to the concept of function as a key piece in analysis, saying that "A function of a variable quantity is an analytic expression formed, in any manner whatever, of the variable quantity and of numbers or constant quantities" (Euler 1748), which is again a clear example of this new approach. The definition and the different notions of function in Euler's work were the key to establishing Mathematical Analysis as a branch of present-day mathematics, as Carmen Martínez analysed in great detail in her article in 2008 (Martínez 2008). 
In particular, the definition given by Euler of trigonometric functions as power series is drawn up in a way completely unrelated to geometry; as a result of this posture, we arrive at the acceptance of complex numbers as a highly powerful instrument in the intrinsic analysis of the functions theory.

While stating ideas of this kind, we can also quote Lagrange who, in his influential work Theory on Analytic Functions (Lagrange 1797), had used series of potentials as functions, also defining that arising at a point simply as the coefficient of the first potential in Taylor's development of the function at that point.

\section{Culmination of work carried out.}

After the analysis made by Fourier, there was a period of 50 years under a context of some concerns regarding the foundations of the analysis, motivated for two main causes. The first was the lack of trust in operations made with infinite series, because it was even not clear if a series of functions converged always or not to the function from which it was obtained. The second cause was the lack of precise definition of the concept of real number that represents the fundamental nucleus for any arithmetization program.

In 1867 Hermann Hankel pointed out that "condition to create a universal arithmetic is that of mathematics purely intellectual, separated from any type of sensitive perception". The total and correct arithmetization of the analysis was only possible when mathematicians understood, as Hankel had already foreseen, that it was needed to consider to real numbers as intellectual structures and not as magnitudes provided intuitively, inherited from Euclidian geometry.

It was in 1872 when many important publications which contributed considerably to the arithmetization of the analysis took place. They culminated half a century of investigations around the idea of function and real number, studies which started in 1822 with the Fourier theory and with an attempt to reduce all the analysis to the arithmetic.

Hugues Charles Méray was one of the mathematicians who publish fast his ideas, considering that he published in 1869 an article (Méray 1869) where underlined a serious reasoning lapse that mathematicians had not realized since the period of Cauchy. The main problem was to define the limit of a succession as a real number and, later, to define in turn a real number as a limit of a succession of rational numbers.

Like Méray, Karl Weierstrass also tried to separate the analysis from the geometry and to base it uniquely on the concept of number. So, Weierstrass presented a refined definition of the concept of limit. The definition of Cauchy made use of expressions like "successive values", "approach indefinitely" or "as small as you want" and, although these expressions are very suggestive, they lack of precision and rigor that nowadays characterize mathematics. His disciple Eduard Heine, based on lessons from Weierstrass, published in 1872 (Heine 
1872) the definition of limit of a function $f(x)$ at $x_{0}$ that, unless notation, is those which usually appears in texts: "Given any $\varepsilon$, if there exists a natural number $\mathrm{n}_{0}$ such that for $0<n<n_{0}$ the difference $f\left(x_{0} \pm n\right)-L$ in absolute value is less than $\varepsilon$, then it is said that $L$ is the limit of $f(x)$ at $x=x_{0}$ ". We can estimate, therefore, a language and a precise and unequivocal symbolism.

In 1871 Georg Cantor had also begun to carry out a arithmetization development similar to those of Meray and Weierstrass. Heine, who knew this work suggested certain simplifications that led to the development of so-called Heine -Cantor, published in 1872 (Heine 1872). The theory presented deals with the convergent successions that do not converge into rational numbers as successions that define irrational numbers.

A completely different approach to the same problem, and one of the most known today, was the one that Richard Dedekind gave that year. It needed to formally construct the real numbers from what is known as Dedekind Cuts (Dedekind 1872). This way, the fundamental theorems on limits could be proved rigorously without using geometric intuition .

\section{The significance of the process}

With arithmetization analysis it was not simply a matter of desgeometrizaring calculation and pointing towards better logical conditions in its foundations: it was, rather, a reduction of different conceptual notions (referring to different objects) to the arithmetical notions. This significantly important process, could only be made from a new abstraction and implicit or explicit introduction of theoretical assumptions about the existence and nature of mathematical entities, as we discussed in the previous section.

It was, moreover, a reductionist intent of the different components with the clear objective of finding a theoretical unity in diversity, whose exposition was demanding a readequacy in the conscience of the nature of the mathematics and even of the own knowledge. So arithmetization analysis can not be considered a mere mechanical and simple process of rigorization of diverse mathematical results, but it must be integrated into a new self-consciousness in the evolution of mathematics.

Consequently, this led to the nineteenth century which was a period in which the advances in mathematics overcame, both in quality and in quantity, the combined production of all earlier times. Furthermore, some prominent schools were created, like the London Mathematical Society (1865), the Société Mathématique de France (1872) and the American Mathematical Society (1888), among others, which led to the implicit social legitimacy of the mathematical job.

In this sense, mathematical rigorization has generated a large set of proof techniques that have enabled a great advance in the scientific production in its different areas. Clearly, in the origins of rigorization, for the mathematicians it was very feasible to know and understand almost all mathematical production, clearly distinguishing those fundamental problems in each area. However, today 
this task becomes impossible since any researcher focuses on knowing and mastering the latest publications of at most two or three areas of mathematical research.

This makes the detection of those most relevant lines of research difficult. Undoubtedly, specialists could agree to point out some important guidelines in their specialty, although the major problems that are studied by the leading researchers are often thought to stand out.

To this difficulty, we add the fact that it is physically impossible for a mathematician, in their daily work to, verify each and every one of the previous results in which it supports his proof. Since the second half of the twentieth century, it is an assumed fact by all that a correct proof is obtained from collective results and, even more, a result is accepted as correct when it finds a consensus among the qualified. In case of any discrepancy between experts, a transcript of the proof based on first-order logic is not used it is resolved by communication and explanation.

This last fact is evident in the appointment of $P$. Davis and $R$. Hersh on the process of verification of the different results that emerge daily in different scientific journals: "Mathematicians in every field rely on each other's work, quote each other; the mutual confidence which permits them to do this is based on confidence in the social system of which they are a part. They do not limit themselves tousing results which they themselves are able to prove from first principles. If a theorem has been published in a respected journal, if the name of the author is familiar, if the theorem has been quoted and used by other mathematicians, then it is considered established. Anyone who has use for it will feel free to do so" (Davis and Hersh, 1982).

\section{Funding}

The authors was partially supported by Vicerrectorado de Investigación, Desarrollo e Innovación de la Universidad de Alicante under project GRE11-23.

\section{References}

- Abel, N. H. (1826). Recherches sur la série $1+\frac{m}{1} x+\frac{m(m-1)}{1 \cdot 2} x^{2}+$ $\frac{m(m-1)(m-2)}{1 \cdot 2 \cdot 3} x^{3}+\cdots$. Oeurres, 1, 219-250.

- Berkeley, G. (1734). The Analyst. London: J. Tonson.

- Bolzano, B. (1817). Rein analytischer beweis des Lehrsatzes, dass zwischen je zwey Werthen, die ein entgegengesetztes Resultat gewähren, wenigstens eine reelle Wurzel der Gleichung liege. Prague: Haase.

- Bombal, F. (2010). Rigor y demostración en matemáticas. Rev. R. Acad. Cienc. Exact. Fís. Nat., 104 (1), 61-79.

- Boyer, C. B. (1986). Historia de la matemática. Madrid: Alianza Editorial.

- Cauchy, A. L. (1821). Cours d'analyse de l'École royale polytechnique. Paris: Imprimerie royale. 
- Cavalieri, B. (1647). Exercitationes geometricae sex. Bologna: Giacomo Monti.

- Davis, P. J. \& Hersh, R. (1982). Experiencia matemática. Barcelona: MEC y Labor.

- Dedekind, R. (1872). Continuity and Irrational Numbers. English translation: From Kant to Hilbert, a Source Book in the Foundations of Mathematics. Oxford: Oxford University Press, 1996, Volume II, 765-779.

- Edwards, C. H. (1979). The Historical Development of the Calculus, Springer-Verlag, New York.

- Euler, L. (1748). Introductio in analysin infinitorum. Lausanne: M.M. Bousquet.

- Ferreirós, J. (2003). Historia. La gaceta de la RSME, 6 (2), 413-436.

- Gauss, C. F. (1963). Disquisitiones arithmeticae. English translation: New Haven, CT: Yale University Press, 1966.

- Hankel, H. (1867). Vorlesungen über die complexen zahlen und ihre functionen. Leipzig: Leopold Voss.

- Heine, E. (1872). Die Elemente der Functionenlehre. J. Reine Angew. Math. 74, 172-188.

- Kline, M. (1992). El pensamiento matemático de la antigüedad a nuestros días. Madrid: Alianza Editorial.

- Lacroix, S. F. (1797). Traité du calcul différentiel et du calcul intégral. París: J.B.M. Duprat.

- Lagrange, J.L. (1797). CEuvres complètes, tome 9.Théorie des fonctions analytiques contenant les principes du calcul différentiel dégagés de toute considération d'infiniment petits, d'évanouissans, de limites et de fluxions, et réduits à l'analyse algébrique des quantités finies. Paris: Imprimerie de la République.

- Mannheim, J. H. (1964). The Genesis of Point Set Topology. Oxford: Pergamon.

- Martínez, C. (2008). El concepto de función en la obra de Euler: un recorrido a través de la constitución del Análisis Matemático Moderno, Miscelánea Matemática, 46, 73-91.

- Méray, H.C. (1869). Remarques sur la nature des quantités définies par la condition de servir de limites à des variables données. Revue des Sociétés savantes, Sci. Mat. Phys. Nat. 2 (4), 280-289.

- Montesinos, V. \& Valdivia, M. (2000). Momentos estelares en el desarrollo de las matemáticas. Real Academia de Ciencias Exactas, Físicas y Naturales, 15-34.

- Ortega, I. (1993). La historia que vivieron los matemáticos. Buenos Aires: Ediciones Letrabuena.

- Rey Pastor, J. \& Babini, J. (1997). Historia de la Matemática. Barcelona: Gedisa.

- Struik, D. J. (1986). A Source Book in Mathematics, 1200-1800. Princeton: Princeton University Press. 


\section{Biography}

L. Segura Abad graduated in 1994 in Mathematics from the Complutense University in Madrid. Since 1998, she has been working at the Department of Mathematical Analysis, University of Alicante. She was manager and speaker at courses and conferences of an educational nature. She is reviewer for some educational journals and author and coauthor of several teaching publications.

J.M. Sepulcre Martínez graduated in Mathematics and Statistical Sciences and Techniques. He received his Ph.D. in 2008 in Mathematics from the University of Alicante (Spain). Since 2007, he has been working at the Department of Mathematical Analysis, University of Alicante. He is author and coauthor of several research papers listed in MathSciNet, some of which have been published in top research journals, and several teaching publications. 